Arq. Bras. Med. Vet. Zootec., v.71, n.1, p.281-290, 2019

\title{
Avaliação do estresse e do desempenho de suínos na fase de creche, empregando-se técnicas de enriquecimento ambiental
}

[Evaluation of stress and performance of pigs during the nursery phase, using environmental enrichment techniques]

\author{
B.M.O. Bezerra ${ }^{1}$, S.S.C. Silva ${ }^{1}$, A.M.A. Oliveira ${ }^{2}$, C.V.O. Silva ${ }^{2}$, R.A. Parente ${ }^{2}$, \\ T.S. Andrade ${ }^{3}$, J.N.B. Evangelista ${ }^{2}$, D.C.S.N. Pinheiro ${ }^{1 *}$
}

${ }^{1}$ Programa de pós-graduação - Universidade Estadual do Ceará - Fortaleza, CE
${ }^{2}$ Faculdade de Veterinária - Universidade Estadual do Ceará - Fortaleza, CE
${ }^{3}$ Programa de pós-graduação - Universidade Federal do Ceará - Fortaleza, CE

\section{RESUMO}

O enriquecimento ambiental é uma ferramenta importante dentro dos sistemas de produção, a fim de promover o bem-estar e favorecer a saúde dos animais. Portanto, o objetivo deste trabalho foi avaliar o efeito do enriquecimento ambiental sobre o estresse de suínos na fase de creche. Foram utilizados 32 leitões, alojados em granja experimental, distribuídos em quatro grupos $(n=8)$ : corda, corrente, garrafa PET e controle negativo. Amostras de sangue foram coletadas no início e no final do experimento para contagem de leucócitos circulantes e determinação de antioxidantes não enzimáticos, óxido nítrico, malondialdeído, e de saliva para avaliação do cortisol. Foi aplicado etograma e fez-a ganho médio de peso diário e a conversão alimentar. Os parâmetros avaliados no primeiro dia do experimento não variaram entre os grupos $(\mathrm{P}>0,05)$. No último dia do experimento, os valores de neutrófilos e da relação neutrófilo/linfócito foram mais elevados nos leitões do grupo corrente, assim como os valores de cortisol salivar $(\mathrm{P}<0,05)$. $\mathrm{O}$ ácido úrico apresentou-se mais elevado nos leitões do grupo corrente e o malondialdeído (MDA) nos do grupo garrafa $(\mathrm{P}<0,05)$. Os enriquecimentos ambientais estimularam comportamentos positivos nos leitões, tendo a corda se destacado como o mais atrativo. Por outro lado, a corrente apresentou efeito negativo sobre a fisiologia dos animais, gerando estresse, assim como a garrafa, que induziu a peroxidação lipídica e um menor ganho de peso nos leitões.

Palavras-chave: suínos, móbiles, parâmetros oxidantes e antioxidantes, fisiologia animal, comportamento animal

\begin{abstract}
Environmental enrichment is an important tool within production systems to promote welfare and animal health. The aim of this study was to evaluate the effect of enrichment objects on stress of piglets in nursery phase. 32 piglets housed in experimental farm were distributed in 4 groups $(n=8)$ : rope, chain, pet bottle and negative control. Blood samples were collected at the beginning and end of the experiment to count circulating leukocytes and determine non-enzymatic antioxidants, nitric oxide, malondialdehyde, and saliva to evaluate cortisol. At the same time, an etogram and evaluation of mean daily gain and feed conversion were applied. The evaluated parameters on first day of the experiment did not vary between groups $(P>0.05)$. On the last day, neutrophil and neutrophil /ymphocyte ratios were higher in chain group piglets, as were salivary cortisol values $(P<0.05)$. Uric acid was higher in chain group and MDA in bottle group $(P<0.05)$. Environmental enrichment stimulated positive behaviors in piglets, where the rope stood out as the most attractive. On the other hand, chain had a negative effect on animal physiology, generating stress, as well as the bottle that induced lipid peroxidation and a lower weight gain in piglets.
\end{abstract}

Keywords: pigs, mobiles, oxidant and antioxidants parameters, animal physiology, animal behavior

Recebido em 11 de agosto de 2017

Aceito em 17 de maio de 2018

*Autor para correspondência (corresponding author)

E-mail: diana.pinheiro@uece.br 


\section{INTRODUÇÃO}

A definição de bem-estar animal é influenciada por padrões morais e éticos das sociedades, o que dificulta o estabelecimento de parâmetros objetivos e quantificáveis para esse termo. De maneira geral, o bem-estar animal pode ser resumido como ausência de sofrimento $(\mathrm{Ohl}$ e Staay, 2012). Nessa perspectiva, na tentativa de trazer condições favoráveis aos animais, foi proposto o enriquecimento ambiental para criar oportunidades comportamentais, expressão de controle sobre o meio e desvio da realização de comportamentos anômalos (Van de Weerd et al., 2006). Nesse sentido, o enriquecimento pode ser utilizado para indicar melhorias sociais, físicas, sensoriais, nutricionais e imunológicas para os animais, de acordo com mudanças realizadas em seus ambientes de confinamento (Newberry, 1995).

O enriquecimento ambiental é utilizado dentro dos sistemas de produção de diferentes espécies, como em frangos de corte (Sans et al., 2014), cabras mestiças em confinamento (Oliveira et al., 2014) e coelhos em gaiolas (Siloto et al., 2009). Desse modo, a escolha do enriquecimento ambiental nos sistemas de produção deve estar de acordo com o efeito desejado sobre os comportamentos dos animais e sua adequação na perspectiva do produtor (Zwicker et al., 2013). Além disso, a introdução do enriquecimento deve ser realizada de maneira cuidadosa, para evitar efeitos negativos no desempenho dos animais que podem comprometer a produção e dificultar a adoção em escala comercial maior. Um exemplo disso é a restrição do ponto de enriquecimento que pode causar competição, agressividade ou agitação nos grupos de animais (Van de Weerd et al., 2006).

O sistema de criação predominante na suinocultura é o intensivo, caracterizado pelo confinamento dos animais, e isso inibe a expressão de comportamento típico da espécie, já que esse ambiente não apresenta estímulos (Foppa et al., 2014). Enriquecimento ambiental dentro desse sistema, como o uso de brinquedos, proporciona bons resultados, principalmente com redução de caudofagia e ociosidade e aumento de interações (Rodarte et al., 2004). No Brasil, a utilização de enriquecimento ambiental por meio de garrafas PET $(500 \mathrm{~mL})$ para leitões, na fase de creche (Campos et al., 2010), e correntes
(Vasconcelos et al., 2015) e brinquedos (Oliveira et al., 2016), na fase de crescimento, mostra-se positiva sobre os comportamentos dos animais. Vale ressaltar que o enriquecimento ambiental atua sobre o sistema imunológico (Luo et al., 2017), o bem-estar fisiológico (Luz et al., 2017) e os índices zootécnicos (Oliveira et al., 2016). Porém, os efeitos desse enriquecimento ainda são pouco estudados sobre a fisiologia animal e, particularmente, sobre os suínos.

Diante do exposto, o objetivo do presente trabalho foi avaliar o efeito do uso de diferentes tipos de enriquecimento ambiental sobre o cortisol, os leucócitos do sangue periférico, os parâmetros do estresse oxidativo e o comportamento de leitões na fase de creche.

\section{MATERIAL E MÉTODOS}

O protocolo experimental foi aprovado pela CEUA/UECE, sob o número 6364557/2015. O trabalho foi desenvolvido no setor de suinocultura da Faculdade de Medicina Veterinária (Favet) da Universidade Estadual do Ceará (UECE), situada em Fortaleza/Ceará, latitude 0378'99', longitude 38 $55^{\prime} 32^{\prime \prime}$ e altitude de $48 \mathrm{~m}$. Essa região é classificada com Aw (tropical quente com inverno seco), de acordo com Köppen e Geiger.

Foram utilizados 32 suínos, 18 machos e 14 fêmeas, distribuídos nos grupos aleatoriamente, do cruzamento Landrace x Large White x Duroc, alojados em baias experimentais de acordo com quatro tratamentos $(n=8)$, em que cada animal foi considerado como uma repetição: grupo corda (GCd), grupo corrente $(\mathrm{GCt})$, grupo garrafa (GG) e grupo controle (GC). Esses móbiles foram disponibilizados durante seis horas por dia, das nove às 15 horas, para leitões no período de creche (45 dias), que estavam alojados em baias suspensas, de piso plástico, onde a corda (tamanho: 1,5m; diâmetro: $20 \mathrm{~mm}$ ) e a corrente (tamanho: 1,5m; diâmetro: $6 \mathrm{~mm}$ ) foram penduradas e fixadas no ponto central das baias, e as duas garrafas PET $(500 \mathrm{~mL})$ foram mantidas livres.

Os leitões foram pesados no dia do desmame (D1), que foi realizado quando os animais estavam com 25 dias de vida, e ao final da fase de creche (D2) (70 dias de vida). Com base nesses dados, foi calculado o ganho médio de 
peso diário (GMPD) (kg/dia) de cada animal. Os leitões foram clinicamente avaliados no primeiro dia do experimento, mediante observação das mucosas, mensuração da temperatura e análise da presença ou ausência de diarreia e/ou tosse. Todos os animais foram considerados saudáveis. $\mathrm{O}$ arraçoamento dos leitões era realizado uma vez ao dia, no início da manhã, quando todos os grupos recebiam a mesma quantidade de ração, ajustada semanalmente. Na primeira semana, foi ofertado 0,2kg/animal/dia (pré-inicial 1); na segunda semana, 0,4kg/animal/dia (pré-inicial 2); na terceira e quarta semanas, $0,7 \mathrm{~kg} / \mathrm{animal} / \mathrm{dia}$ (inicial 1); e na quinta e sexta semanas, 0,9kg/animal/dia (inicial 2). Com base no consumo de ração dos animais e do ganho de peso, foi obtido o valor de conversão alimentar (CA).

Foram realizadas coletas de sangue por venopunção jugular nos animais no início do experimento (D1) e no final (D45), no momento de saída da creche. As amostras sanguíneas foram coletadas em tubos com anticoagulante (EDTA) e sem anticoagulante e transportadas até o laboratório. No laboratório, foram quantificados leucócitos totais $\left(\times 10^{3} / \mu \mathrm{L}\right) \mathrm{em}$ aparelho de automação hematológico, e o diferencial de leucócitos foi realizado por meio de leitura de esfregaço sanguíneo corado com panótico rápido comercial, por patologista clínico veterinário $(400 x)$, para determinação de neutrófilos $\left(\mathrm{x} 10^{3} / \mu \mathrm{L}\right)$, linfócitos $\left(\mathrm{x} 10^{3} / \mu \mathrm{L}\right)$ e relação neutrófilo/linfócito.

As amostras de sangue coletadas em tubos sem anticoagulante foram centrifugadas (3.000rpm/10 minutos) para obtenção do soro. No soro, foram dosados os teores de albumina $(\mathrm{g} / \mathrm{dL})$, ácido úrico $(\mathrm{mg} / \mathrm{dL})$, bilirrubina total $(\mathrm{mg} / \mathrm{dL})$, utilizando-se metodologias específicas conforme o fabricante dos kits comerciais, em analisador bioquímico automatizado.

O óxido nítrico $(\mathrm{ON})$ sérico foi mensurado por meio da metodologia de Griess modificada em placa de ELISA (D’Ávila et al., 2008). Para tanto, o reagente de Griess preparado $(100 \mu \mathrm{L})$ foi adicionado a $50 \mu \mathrm{L}$ do soro e a $50 \mu \mathrm{L}$ de água destilada em cada poço da placa, para obtenção dos valores de nitrito em espectrofotômetro (550nm). Para obtenção das quantidades de proteínas séricas, foi utilizado o reagente de Bradford $(200 \mu \mathrm{L})$ adicionado a $4 \mu \mathrm{L}$ do soro em cada poço da placa, e a leitura foi realizada em espectrofotômetro (600nm) (Bradford, 1976). Os resultados foram expressos em $\mu \mathrm{g}$ nitrito/ $\mu \mathrm{g}$ proteína.

Para avaliação da peroxidação lipídica, foi realizada a quantificação do malondialdeído (MDA) sérico em tubos de vidro, onde foram adicionados $250 \mu \mathrm{L}$ de soro, seguido por $400 \mu \mathrm{L}$ de ácido perclórico (35\%) em banho-maria $\left(37^{\circ} \mathrm{C}\right.$; uma hora). A mistura foi centrifugada ( $1400 \mathrm{~g} ; 10$ minutos) e os $600 \mu \mathrm{L}$ do sobrenadante foram adicionados a $200 \mu \mathrm{L}$ de ácido tiobarbitúrico a 1,2\%. Essa mistura foi levada ao banho-maria $\left(95^{\circ} \mathrm{C} ; 30\right.$ minutos). Após resfriada, a leitura foi realizada em espectrofotômetro (535nm). Os resultados obtidos foram expressos em nmol/mL de soro (Draper e Hadley 1990).

A saliva dos leitões foi coletada no final do experimento (D45), com auxílio de cotonetes longos estéreis, que foram introduzidos no interior da cavidade oral dos animais, para que fossem mastigados até o maço de algodão ficar umedecido. Posteriormente, a saliva foi extraída por pressão manual do algodão para microtubos, centrifugadas (1.500rpm/10min) e armazenadas em temperatura de $-20^{\circ} \mathrm{C}$ até o processamento. Os sobrenadantes foram analisados em equipamento automatizado por meio do método de quimioluminescência.

Durante os 45 dias da creche, foram avaliados e anotados os comportamentos dos leitões, em dias alternados. No período das nove às 15 horas, de 10 em 10 minutos, o mesmo observador, localizado em ponto fixo em frente às baias, anotava quantos animais expressavam cada tipo de comportamento, descrito na Tab. 2. Esse modelo de etograma foi baseado e adaptado de Campos et al. (2010).

Os resultados foram expressos em média \pm desvio-padrão e foram considerados significativos com $\mathrm{P} \leq 0,05$. Os dados foram submetidos ao teste de Kruskal-Wallis, seguido de Dunn (Graphpad Prism®). A correlação de Spearman (Graphpad Prism®) foi utilizada para comparação de dados. Os comportamentos foram expressos em porcentagem (\%). 
Tabela 1. Variáveis de desempenho de leitões na fase de creche submetidos a enriquecimento ambiental

\begin{tabular}{|c|c|c|c|c|}
\hline \multirow[b]{2}{*}{$\begin{array}{l}\text { Variáveis de } \\
\text { Desempenho }\end{array}$} & \multicolumn{4}{|c|}{ Enriquecimento Ambiental } \\
\hline & Corda & Corrente & Garrafa & Controle \\
\hline Peso Inicial (kg) & $5,28 \pm 0,83^{a}$ & $4,88 \pm 1,09^{\mathrm{a}}$ & $4,86 \pm 1,02^{\mathbf{a}}$ & $5,59 \pm 0,48^{\mathbf{a}}$ \\
\hline Peso Final (kg) & $22,77 \pm 2,39^{a}$ & $23,06 \pm 2,30^{\mathbf{a b}}$ & $20,8 \pm 2,28^{\mathbf{a}}$ & $27,08 \pm 1,03^{\mathbf{b}}$ \\
\hline GPMD (kg) & $0,39 \pm 0,04^{\mathbf{a}}$ & $0,40 \pm 0,03^{\mathbf{a b}}$ & $0,35 \pm 0,03^{\mathbf{a}}$ & $0,48 \pm 0,01^{\mathbf{b}}$ \\
\hline $\mathrm{CA}$ & $1,53 \pm 0,16^{\mathbf{a}}$ & $1,47 \pm 0,12^{\mathbf{a b}}$ & $1,68 \pm 0,15^{\mathbf{a}}$ & $1,24 \pm 0,03^{\mathbf{b}}$ \\
\hline
\end{tabular}

Letras diferentes na mesma linha denotam diferença significativa $(\mathrm{p}<0,05)$.

Tabela 2. Padrões de comportamento avaliados em etograma aplicado

\begin{tabular}{|c|c|}
\hline Comportamento & Descrição* \\
\hline Comportamento agonístico & Animal brigando, mordendo ou arranhando o outro com os dentes. \\
\hline Fuçando a baia & $\begin{array}{l}\text { Animal fuçando o piso da baia, as laterais ou ao redor do comedouro } \\
\text { com o focinho. }\end{array}$ \\
\hline Fuçando o outro & Animal fuçando a orelha, a cauda ou a barriga do outro com o focinho. \\
\hline Dormindo ou deitado & $\begin{array}{l}\text { Animal deitado com o corpo em contato com o piso ou estirado sobre } \\
\text { ele, com olhos fechados ou abertos. }\end{array}$ \\
\hline Ingerindo alimento ou água & $\begin{array}{l}\text { Animal ingerindo alimento (ração ou água) no comedouro ou no } \\
\text { bebedouro. }\end{array}$ \\
\hline Locomovendo-se & Animal em movimento de caminhada pela baia. \\
\hline Outros & $\begin{array}{l}\text { Animal sentado (apoiado com a parte posterior e as patas dianteiras no } \\
\text { piso); animal parado sobre o piso, apoiado nas quatro patas e sem } \\
\text { nenhum movimento aparente ou animal excretando dejetos (fezes e } \\
\text { urina). }\end{array}$ \\
\hline Brincando entre eles & Animal correndo dentro da baia ou animal apoiado sobre o outro. \\
\hline Brincado com os objetos & Animal fuçando, abocanhando ou empurrando os objetos. \\
\hline
\end{tabular}

\section{RESULTADOS E DISCUSSÃO}

A importância deste estudo se deve ao fato de o enriquecimento ambiental ser uma alternativa relevante no sistema de produção, para diminuir a inatividade dos animais, proporcionar bemestar animal e melhorar seu potencial produtivo (Andrade et al., 2015), que pode ser avaliado por meio de parâmetros de desempenho. Os valores relacionados aos desempenhos peso inicial, peso final, ganho de peso médio diário (GPMD) e conversão alimentar encontram-se na Tab. 1 Nesta, pode-se observar que, no início do experimento, não havia diferença $(\mathrm{P}>0,05)$ entre os pesos do animais entre os tratamentos, porém, no final dele, o GC apresentou o maior ganho de peso e a menor conversão alimentar quando comparado ao GCd e ao GG $(\mathrm{P}<0,05)$. A corda e a garrafa foram os objetos mais atrativos para os leitões, levando-os a desenvolverem mais comportamentos ativos e, assim, aumentarem o exercício físico e o gasto de energia, que, consequentemente, levam a um menor ganho de peso corporal (Vieira et al., 2015). Os animais do GCt não apresentaram diferença nas variáveis de desempenho avaliadas em relação ao GC, o que pode ser justificado pelo fato de esse objeto não estimular tanta atividade física dos animais. No estudo de Oliveira et al. (2014), brinquedos pendurados melhoraram o desempenho de crescimento de leitões na fase de creche, o que não foi observado no presente trabalho.

Os parâmetros sanguíneos referentes ao primeiro dia do experimento (D1) não variaram entre os grupos estudados e encontram-se nas Tab. 3 e 4. Em D45, há algumas diferenças entre os grupos, que serão discutidas a seguir:

Os leucócitos são células de defesa e têm uma forte relação entre saúde e doença. Neste estudo, foi focalizado o comportamento animal e sua repercussão sobre os leucócitos circulantes, cujos dados encontram-se na Tab. 3. Os valores de 
leucócitos totais e linfócitos não variaram $(\mathrm{P}>0,05)$ entre os tratamentos. Os neutrófilos e, consequentemente, a relação neutrófilo/linfócito apresentaram-se mais elevados nos leitões do GCt quando comparados aos grupos GG e GC $(\mathrm{P}<0,05)$ e não apresentaram diferença em relação ao GCd $(\mathrm{P}>0,05)$ (Tab. 3). Esses resultados podem ser associados ao fato de a corrente ser um objeto fixo não destrutível, que pode acabar frustrando os animais em não expressar os comportamentos tão importantes para essa espécie, como fuçar, mastigar e destruir o objeto, levando ao estresse e provocando um mecanismo compensatório com o aumento em número de neutrófilos circulantes e da relação neutrófilo/linfócito. Vale ressaltar que os suínos, fisiologicamente, apresentam mais linfócitos do que neutrófilos circulantes e que o estresse por esteroides causa neutrofilia e linfopenia (Stockham e Scott, 2011), corroborando os achados do presente estudo.

Tabela 3. Parâmetros fisiológicos em leitões na fase de creche submetidos a enriquecimento ambiental

\begin{tabular}{|c|c|c|c|c|c|c|c|c|}
\hline \multirow[b]{3}{*}{$\begin{array}{l}\text { Parâmetros } \\
\text { Fisiológicos }\end{array}$} & \multicolumn{8}{|c|}{ Enriquecimento ambiental } \\
\hline & \multicolumn{2}{|c|}{ Corda } & \multicolumn{2}{|c|}{ Corrente } & \multicolumn{2}{|c|}{ Garrafa } & \multicolumn{2}{|c|}{ Controle } \\
\hline & D1 & D45 & D1 & D45 & D1 & D45 & D1 & D45 \\
\hline $\begin{array}{l}\text { Leucócitos } \\
\left(\mathrm{x} 10^{3} / \mu \mathrm{L}\right)\end{array}$ & $10,6 \pm 2,3^{\mathrm{a}}$ & $16,7 \pm 3,5^{\mathrm{a}}$ & $15,7 \pm 4,4^{\mathrm{a}}$ & $18,2 \pm 2,7^{\mathrm{a}}$ & $11,5 \pm 3,4^{\mathrm{a}}$ & $15,4 \pm 1,8^{\mathrm{a}}$ & $13,4 \pm 2,1^{\mathrm{a}}$ & $17,1 \pm 2,7^{\mathrm{a}}$ \\
\hline $\begin{array}{l}\text { Neutrófilos } \\
\left(\mathrm{x} 10^{3} / / \mu \mathrm{L}\right)\end{array}$ & $3,8 \pm 2,6^{\mathrm{a}}$ & $4,5 \pm 1,0^{\mathrm{ab}}$ & $5,6 \pm 2,2^{\mathrm{a}}$ & $6,6 \pm 1,2^{a}$ & $4,3 \pm 2,8^{\mathrm{a}}$ & $3,7 \pm 1,1^{b}$ & $4,3 \pm 1,6^{\mathrm{a}}$ & $3,8 \pm 1,1^{\mathrm{b}}$ \\
\hline $\begin{array}{l}\text { Linfócitos } \\
\left(\mathrm{x} 10^{3} / / \mu \mathrm{L}\right)\end{array}$ & $6,9 \pm 0,6^{\mathrm{a}}$ & $11,8 \pm 3,9^{\mathrm{a}}$ & $9,7 \pm 2,3^{\mathrm{a}}$ & $11,3 \pm 2,2^{\mathrm{a}}$ & $7,2 \pm 1,7^{\mathrm{a}}$ & $11,3 \pm 1,5^{\mathrm{a}}$ & $9,0 \pm 1,1^{\mathrm{a}}$ & $13,0 \pm 2,1^{\mathrm{a}}$ \\
\hline Relação N/L & $0,4 \pm 0,1^{\mathrm{a}}$ & $0,43 \pm 0,2^{\mathrm{ab}}$ & $0,5 \pm 0,1^{\mathrm{a}}$ & $0,61 \pm 0,1^{\mathrm{a}}$ & $0,5 \pm 0,2^{\mathrm{a}}$ & $0,33 \pm 0,1^{\mathrm{b}}$ & $0,5 \pm 0,2^{\mathrm{a}}$ & $0,30 \pm 0,1^{\mathrm{b}}$ \\
\hline $\begin{array}{l}\text { Cortisol salivar } \\
(\mathrm{ng} / \mathrm{mL})\end{array}$ & $*$ & $2,15 \pm 0,2^{\mathrm{ab}}$ & * & $3,36 \pm 1,0^{\mathrm{a}}$ & $*$ & $2,33 \pm 0,5^{\mathrm{ab}}$ & $*$ & $1,93 \pm 0,1^{\mathrm{b}}$ \\
\hline
\end{tabular}

Letras diferentes entre os grupos no mesmo dia de coleta denotam diferença significativa $(\mathrm{P}<0,05)$.

Relação N/L: N=neutrófilos, L=linfócitos. D1: primeiro dia do experimento; D45: último dia do experimento.

*Cortisol salivar não foi dosado no primeiro dia do experimento.

$\mathrm{Na}$ tentativa de avaliar a influência do enriquecimento ambiental sobre o estresse dos leitões, foi determinado o cortisol salivar, cujos valores encontram-se na Tab. 3. O cortisol salivar apresentou-se mais elevado nos leitões de GCt quando comparados aos do GC $(\mathrm{P}<0,05)$ (Tab. 3). Este fato pode estar associado a uma situação de estresse para esses animais, como relatado anteriormente, pois a corrente representa um objeto rígido; inclusive a relação neutrófilo/linfócito apresentou correlação positiva com o cortisol nos animais desse grupo (Tab. 4). Esses dados corroboram com os resultados obtidos em suínos alojados em ambientes enriquecidos, nos quais os animais apresentaram maiores concentrações basais de cortisol (De Jong et al., 1998). O cortisol é um mediador hormonal esteroide produzido pelas glândulas suprarrenais e está diretamente envolvido no controle do estresse pelo organismo (Stockham e Scott, 2011). Por isso, esse parâmetro é bastante utilizado em protocolos em que o estresse é avaliado, uma vez que o cortisol resulta da atividade do eixo hipotálamo-hipófiseadrenal. Então, quando um animal é submetido a uma situação estressante, há uma ativação desse eixo e, consequentemente, o aumento do cortisol (Escribano et al., 2012).

Tabela 4. Correlação entre cortisol salivar, relação N/L e ácido úrico dos leitões do grupo corrente

\begin{tabular}{ccccc}
\hline & & Relação N/L & Cortisol & Ácido úrico \\
\hline \multirow{2}{*}{ Relação N/L } & $\mathrm{r}$ & - & 0,5274 & $-0,3179$ \\
& $\mathrm{p}$ & - & $<0,001$ & 0,0013 \\
\multirow{2}{*}{ Cortisol } & $\mathrm{r}$ & & - & $-0,3178$ \\
& $\mathrm{p}$ & & - & 0,0014 \\
\hline
\end{tabular}

r: coeficiente de correlação; p: diferença significativa. 
Para manutenção da homeostase dos organismos, é necessário o equilíbrio entre o sistema oxidante e o antioxidante, pois a geração das espécies reativas de oxigênio (EROS) provoca danos que podem ser evitados por meio da atuação dos antioxidantes (Sies e Stahl, 1995). Neste trabalho, os teores de antioxidantes não enzimáticos foram medidos e encontram-se na Tab. 5. Os antioxidantes não enzimáticos plasmáticos, albumina e bilirrubina não apresentaram diferença significativa entre os grupos de acordo com os enriquecimentos ambientais $(\mathrm{P}>0,05)$, enquanto o ácido úrico apresentou-se mais elevado nos leitões de GCt $(\mathrm{P}<0,05)$ (Tab. 5). Esse dado pode ser explicado por uma compensação antioxidante do organismo desses leitões, a qual aumenta o ácido úrico para neutralizar o aumento na produção de radicais livres induzido pelo estresse desencadeado pelo uso do objeto. O ácido úrico é o mais importante antioxidante não enzimático, seguido pelo complexo bilirrubina-albumina, que, em concentrações fisiológicas, atua contra a oxidação mediada por radicais livres na corrente sanguínea, inibindo reações nitrosativas extracelulares (Mancuso et al., 2006). O ácido úrico apresentou correlação negativa com o cortisol e com a relação neutrófilo/linfócito no grupo corrente (Tab. 4), e correlação positiva com o GMPD do GG (Tab. 6). O ácido úrico é o produto final de oxidação do catabolismo das purinas e seus níveis séricos dependem de fatores metabólicos (Marion et al., 2011), o que pode ser associado a sua correlação positiva com o GMPD. E sua correlação negativa com o cortisol e com a relação neutrófilo pode ser relacionada ao consumo desse antioxidante devido ao aumento na produção de radicais livres gerada pelo estresse

Tabela 5. Parâmetros fisiológicos em leitões na fase de creche submetidos a enriquecimento ambiental

\begin{tabular}{|c|c|c|c|c|c|c|c|c|}
\hline \multirow{3}{*}{$\begin{array}{c}\text { Parâmetros } \\
\text { Fisiológicos }\end{array}$} & \multicolumn{8}{|c|}{ Enriquecimento ambiental } \\
\hline & \multicolumn{2}{|c|}{ Corda } & \multicolumn{2}{|c|}{ Corrente } & \multicolumn{2}{|c|}{ Garrafa } & \multicolumn{2}{|c|}{ Controle } \\
\hline & D1 & D45 & D1 & D45 & D1 & D45 & D1 & D45 \\
\hline $\begin{array}{l}\text { Albumina } \\
(\mathrm{g} / \mathrm{dL})\end{array}$ & $3,2 \pm 0,2^{\mathrm{a}}$ & $3,0 \pm 0,3^{\mathrm{a}}$ & $3,0 \pm 0,6^{\mathrm{a}}$ & $3,0 \pm 0,3^{\mathrm{a}}$ & $3,0 \pm 0,2^{\mathrm{a}}$ & $3,06 \pm 0,68^{\mathrm{a}}$ & $3,3 \pm 0,2^{\mathrm{a}}$ & $3,59 \pm 0,58^{\mathrm{a}}$ \\
\hline $\begin{array}{l}\text { Bilirrrubina } \\
\text { (mg/dL) }\end{array}$ & $0,4 \pm 0,1^{\mathrm{a}}$ & $0,4 \pm 0,2^{\mathrm{a}}$ & $0,4 \pm 0,2^{\mathrm{a}}$ & $0,4 \pm 0,2^{\mathrm{a}}$ & $0,4 \pm 0,1^{\mathrm{a}}$ & $0,39 \pm 0,13^{\mathrm{a}}$ & $0,5 \pm 0,3^{\mathrm{a}}$ & $0,53 \pm 0,29^{\mathrm{a}}$ \\
\hline $\begin{array}{l}\text { Ácido úrico } \\
(\mathrm{mg} / \mathrm{dL})\end{array}$ & $0,4 \pm 0,2^{\mathrm{a}}$ & $0,2 \pm 0,05^{\mathrm{ab}}$ & $0,3 \pm 0,1^{\mathrm{a}}$ & $0,3 \pm 0,05^{\mathrm{a}}$ & $0,3 \pm 0,2^{\mathrm{a}}$ & $0,20 \pm 0,05^{\mathrm{ab}}$ & $0,3 \pm 0,2^{\mathrm{a}}$ & $0,19 \pm 0,06^{b}$ \\
\hline$(\mu \mathrm{g} \mathrm{ONO} / \mu \mathrm{g} \mathrm{pt})$ & $0,08 \pm 0,02^{\mathrm{a}}$ & $0,06 \pm 0,02^{\mathrm{a}}$ & $0,07 \pm 0,03^{\mathrm{a}}$ & $0,04 \pm 0,01^{\mathrm{a}}$ & $0,07 \pm 0,03^{\mathrm{a}}$ & $0,06 \pm 0,02^{\mathrm{a}}$ & $0,06 \pm 0,04^{\mathrm{a}}$ & $0,04 \pm 0,02^{a}$ \\
\hline $\begin{array}{c}\text { MDA } \\
\text { (nmol/ml soro) }\end{array}$ & $8,2 \pm 2,3^{\mathrm{a}}$ & $10,0 \pm 1,2^{\mathrm{ab}}$ & $9,7 \pm 2,6^{\mathrm{a}}$ & $7,6 \pm 1,3^{\mathrm{a}}$ & $12,6 \pm 4,0^{\mathrm{a}}$ & $12,79 \pm 2,61^{\mathrm{b}}$ & $12,8 \pm 5,8^{\mathrm{a}}$ & $8,18 \pm 2,10^{b}$ \\
\hline
\end{tabular}

Letras diferentes entre os grupos no mesmo dia de coleta denotam diferença significativa $(\mathrm{P}<0,05)$.

ON: óxido nítrico; ONO: nitrito; pt: proteína total sérica.

D1: primeiro dia do experimento; D45: último dia do experimento.

Dentre os EROS, destaca-se o ON, que é produzido por diversas células de defesa e pelo endotélio vascular. Os níveis séricos de ON encontram-se na Tab. 5. Os leitões não apresentaram mudanças na produção desse potente radical livre, de acordo com os enriquecimentos ambientais utilizados em suas baias $(\mathrm{P}>0,05)$. ON é um radical livre produto da reação de oxirredução da L-arginina em Lcitrulina, que acontece nos macrófagos, sendo esse agente importante da defesa do organismo contra patógenos (Marletta et al., 1988). Quando ele é produzido em excesso, causa danos oxidativos em proteínas, bem como a peroxidação lipídica, podendo levar à citotoxicidade e a dano celular (Szabó, 2003).
Para avaliação da peroxidação lipídica, os níveis de MDA foram mensurados e encontram-se na Tab. 5. MDA apresentou-se mais elevado nos leitões do GG em comparação ao GCt $(\mathrm{P}<0,05)$. Esse aumento do MDA observado neste protocolo experimental pode ser associado ao fato de a garrafa ser um objeto móvel e estimular a movimentação dos animais nas baias. Dados semelhantes foram observados em humanos realizando exercício físico onde havia estimulação da lipoperoxidação (Schneider e Oliveira, 2004). Esse processo é um dos principais mecanismos moleculares decorrentes do dano oxidativo, atuando sobre a membrana celular, o que gera toxicidade, seguida por dano tecidual (Dianzani e Barrera, 2008). A 
peroxidação lipídica resulta em uma variedade de intermediários e produtos finais, como o MDA (Moselhy et al., 2013). Apesar de o ON não ter variado entre os grupos, ele apresentou correlação positiva com o MDA (Tab. 6), demonstrando que esse radical livre está relacionado à peroxidação lipídica, porém não aumentou significativamente nos leitões do GG. Esse fato pode estar associado à existência de outros radicais na corrente sanguínea (Sies e Stahl, 1995), os quais não foram mensurados no presente estudo. Por outro lado, o MDA apresentou correlação positiva com o GMPD dos animais do GG, demostrando que o esforço físico também pode ter influenciado esse parâmetro de desempenho, uma vez que, com o aumento do MDA, houve uma redução do GMPD (Tab. 6).

Tabela 6. Correlação entre MDA, GMPD, ácido úrico e óxido nítrico dos leitões do grupo garrafa

\begin{tabular}{|c|c|c|c|c|c|}
\hline & & GMPD & MDA & Ácido úrico & Óxido nítrico \\
\hline \multirow{2}{*}{ GMPD } & $\mathrm{r}$ & - & 0,5485 & 0,5270 & $-0,3144$ \\
\hline & $\mathrm{p}$ & - & $<0,001$ & $<0,001$ & 0,014 \\
\hline \multirow{2}{*}{ MDA } & $\mathrm{r}$ & & - & 0,5061 & 0,5497 \\
\hline & $\mathrm{p}$ & & - & $<0,001$ & $<0,001$ \\
\hline \multirow{2}{*}{ Ácido úrico } & $\mathrm{r}$ & & & - & 0,8243 \\
\hline & $\mathrm{p}$ & & & - & $<0,001$ \\
\hline
\end{tabular}

r: coeficiente de correlação; p: diferença significativa.

Além dos parâmetros fisiológicos avaliados, decidiu-se pela aplicação de etograma para avaliar a influência dos móbiles sobre o comportamento animal. Os resultados da influência do enriquecimento ambiental sobre o comportamento de leitões estão apresentados na Tab. 7. Entre os comportamentos avaliados, os mais expressos foram dormindo/deitado $\mathrm{e}$ comendo/bebendo, tendo o GC apresentado maior porcentagem do comportamento dormindo/deitado quando comparado aos outros grupos (Fig. 1). Esse dado pode estar relacionado com o maior GMPD dos animais desse grupo, que pode ser atribuído ao longo tempo de permanência em repouso e ao consequente menor gasto de energia (Vieira et al., 2015).

Tabela 7. Comportamentos de leitões em fase de creche avaliados por etograma

\begin{tabular}{lcccc}
\multicolumn{1}{c}{$\begin{array}{c}\text { Comportamentos } \\
\text { (Porcentagem/\%) }\end{array}$} & Corda & Corrente & Garrafa & Controle \\
\cline { 2 - 5 } Mordendo & 0 & 0,3 & 0,1 & 0 \\
Fuçando a baia & 6,9 & 7,0 & 5,6 & 3,8 \\
Fuçando o outro & 1,6 & 2,1 & 1,3 & 5,0 \\
Dormindo/deitado & 42,7 & 34,1 & 33,4 & 61,5 \\
Comendo/bebendo & 35,2 & 45,4 & 46,10 & 25,0 \\
Locomovendo-se & 1,7 & 2,8 & 3,8 & 1,9 \\
Parado/excretando & 0,4 & 0,8 & 0,9 & 2,4 \\
Brincando entre si & 0,2 & 0,4 & 0,2 & 0,4 \\
Brincando com objeto & 11,3 & 7,1 & 8,6 & 0 \\
\multicolumn{1}{c}{ Total } & 100 & 100 & 100 & 100 \\
\hline
\end{tabular}

Muitas são as tentativas de introdução de enriquecimento ambiental dentro do sistema de produção para estimular comportamentos positivos nos animais. Porém, a incerteza do que realmente é atrativo para cada espécie pode impossibilitar o uso dele. Mediante o exposto, o móbile mais atrativo para leitões na fase de creche foi a corda, seguido pela garrafa e pela corrente (Fig. 1). Os leitões alojados em baias enriquecidas apresentaram maior porcentagem de comportamento exploratório, como o de fuçando a baia e o da interação com os móbiles, do que os do grupo controle (Fig. 1). O uso de enriquecimento ambiental em longo prazo induz o comportamento exploratório em suínos (Zwicker et al., 2013). Já o comportamento fuçando o outro foi mais expresso nos leitões do GC $(5,0 \%)$, o que pode ser um fator de estimulação de brigas. Tal melhora no comportamento dos animais alojados em 
ambiente enriquecido já foi observada anteriormente em estudo com brinquedos suspensos em leitões no período de creche.Essa

\section{Corda}

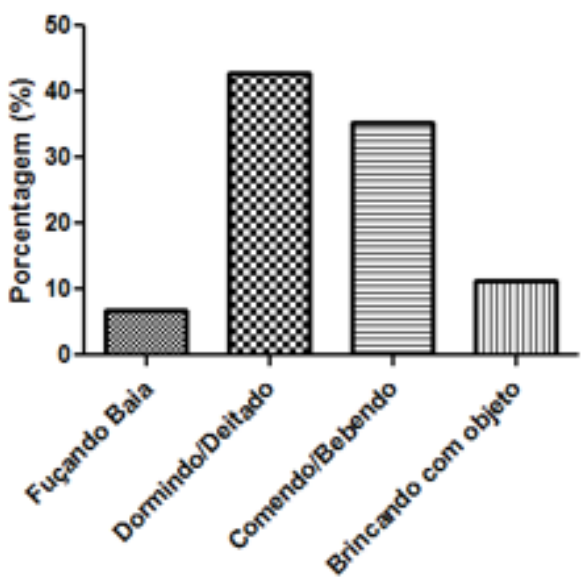

Garrafa

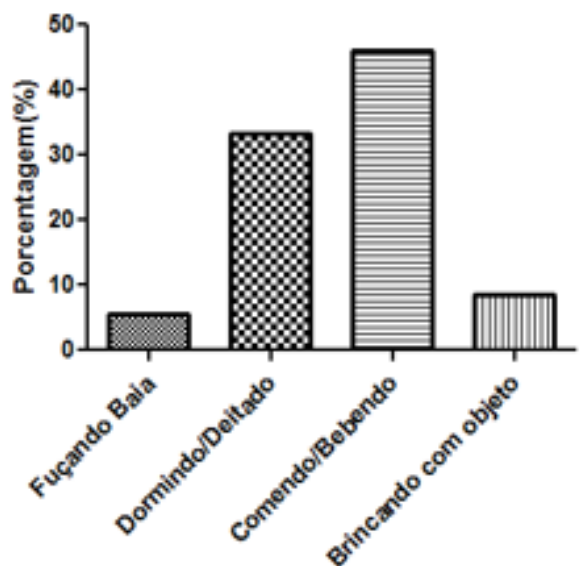

técnica pode ser uma ferramenta importante na promoção de bem-estar na suinocultura (Oliveira et al., 2016).

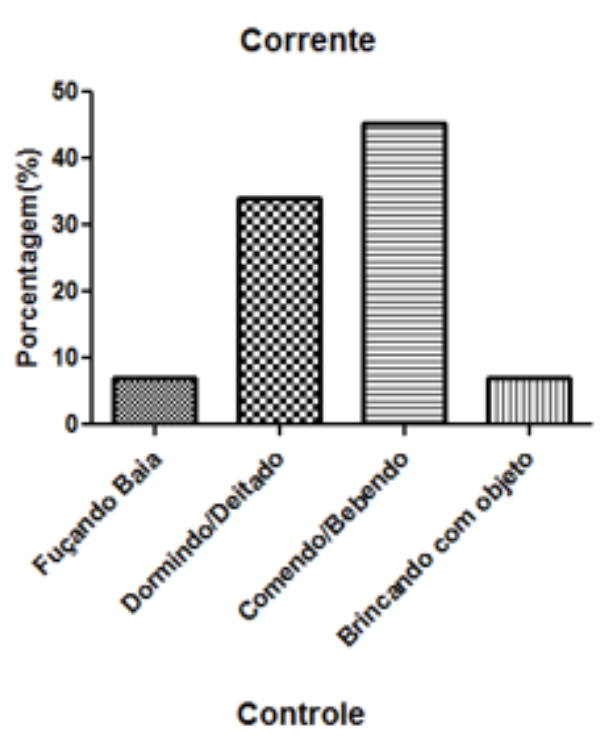

Figura 1. Principais comportamentos de leitões em fase de creche avaliados por etograma.

\section{CONCLUSÃO}

Neste trabalho, o enriquecimento ambiental estimulou comportamentos positivos nos leitões, tendo a corda se destacado como o mais atrativo. Por outro lado, a corrente apresentou efeito negativo sobre a fisiologia dos animais, gerando estresse, assim como a garrafa, que induziu a peroxidação lipídica e um menor ganho de peso nos leitões. Dessa forma, conclui-se que o enriquecimento ambiental melhora os comportamentos dos leitões, porém pode provocar alterações fisiológicas, o que ressalta a importância do estudo dessa técnica antes da aplicação nos sistemas de produção.

\section{AGRADECIMENTOS}

À Coordenação de Aperfeiçoamento de Pessoal de Nível Superior (Capes) e à Financiadora de Estudos e Projetos (Finep), pelo suporte financeiro a este trabalho. 


\section{REFERÊNCIAS}

ANDRADE, T.V.; SOUSA, P.H.A.A.; BARROS JÚNIOR, C.P. et al. Aspectos relacionados ao bem-estar animal na produção de suínos. $J$. Anim. Behav. Biometeorol, v.3, p.124-127, 2015.

BRADFORD, M.M. A rapid and sensitive method for the quantitation of microgram quantities of protein utilizing the principle of protein-dye binding. Anal. Biochem, v.72, p.248254, 1976.

CAMPOS, J.A.; TINÔCO, I.F.F.; SILVA, F.F. et al. Enriquecimento ambiental para leitões na fase de creche advindos de desmame aos 21 e 28 dias. Rev. Bras. Cienc. Agrar., v.5, p.272-278, 2010.

D’ÁVILA, V.G.F.C.; SOUSA JÚNIOR, N.B.; SOUSA, F.B. et al. Avaliação da produção de óxido nítrico em ratos, submetidos aos exercícios aeróbios e anaeróbios. Rev. Bras. Cienc. Farm., v.44, p.755-761, 2008.

DE JONG, I.C.; EKKEL, E.D.; DE BURGWAL, J.A.D. et al. Effects of strawbedding on physiological responses to stressors and behavior in growing pigs. Physiol. Behav., v.64, p.303310, 1998.

DIANZANI, M.; BARRERA, G. Pathology and physiology of lipid peroxidation and its carbonyl products. In: ÁLVAREZ, S.; EVELSON, P. Free Radical Pathophysiology. Kerala: Transworld Research Network, 2008, p. 19-38.

DRAPER, H.H.; HADLEY, M. Malondialdehyde determination as index of lipid peroxidation. Methods Enzymol., v.186, p.421431,1990

ESCRIBANO, D.; FUENTES-RUBIO, M.; CERÓN, J.J. Validation of an automated chemiluminescent immunoassay for salivary cortisol measurements in pigs. J. Vet. Diag. Invest., v.24, p.918-923, 2012.

FOPPA, L.; CALDARA, F.R.; MACHADO, S.P. et al. Enriquecimento ambiental e comportamento de suínos: revisão. Braz. J. Bioscis. Engin., v.8, p.1-7, 2014.

LUO, L.; GEERS, R.; REIMERT, I. et al. Effects of environmental enrichment and regrouping on natural autoantibodies-binding danger and neural antigens in healthy pigs with different individual characteristics. Animal, v.11, p.1-8, 2017.
LUZ, C.S.M.; FARIAS, L.A.; PIMENTA, J.L.L.A. et al. Physiological parameters of pigs raised with and without environmental enrichment. J. Agric. Sci., v.9, p.176-185, 2017.

MANCUSO, C.; BONSIGNORE, A.; CAPONE, C. et al. Albumin-bound bilirubin interacts with nitric oxide by a redox mechanism. Antioxid. Redox Signal., v.8, p.487-494, 2006.

MARION, M.; CARVALHO, J.A.M.; BOCHI, G.V. et al. Ácido úrico como fator de risco para doenças cardiovasculares e síndrome metabólica. Rev. Bras. Farm., v.92, p.3-8, 2011.

MARLETTA, M.A.; YOON, P.S.; IYENGAR, R. et al. Macrophague oxidation of L-arginine to nitrite and nitrate: nitric oxide is an intermediate. Biochemistry, v.27, p.8706-8711, 1988.

MOSELHY, H.F.; REID, R.G.; YOUSEF, S. et al. A specific, accurate, and sensitive measure of total plasma malondialdehyde by HPLC. J. Lip. Res., v.54, p.852-858, 2013.

NEWBERRY, R.C. Environmental enrichment: increasing the biological relevance of captive environments. Appl. Anim. Behav. Sci., v.44, p.229-243. 1995.

OHL, F.; STAAY, F.J. Animal welfare: at the interface between science and society. Vet. J., v.192, p.13-19, 2012.

OLIVEIRA, A.P.G.; COSTA, W.M.; COSTA, W.M. et al. Influência do enriquecimento ambiental nos padrões de comportamentos sociais e anormais de cabras em confinamento. Arch. Vet. Sci., v.19, 7p., 2014.

OLIVEIRA, R.F.; SOARES, R.T.R.N.; MOLINO, J.P. et al. Environmental enrichment improves the performance and behavior of piglets in the nursery phase. Arq. Bras. Med. Vet. Zootec., v.68, p.415-421, 2016.

RODARTE, L.F.; DUCOING, A.; GALINDO, F. et al. The effect of environmental manipulation on behavior, salivary cortisol and growth of piglets weaned at 14 days of age. $J$. Appl. Anim. Welf. Sci., v.7, p.171-179, 2004.

SANS, E.C.O; $\quad$ FEDERICI, J.F.; HAMMERSCHMIDT, J. et al. O enriquecimento ambiental sobre o bem-estar de frangos de corte. Cienc. Rural, v.44, p.1867-1873, 2014. 
SCHNEIDER, C.D.; OLIVEIRA, A.R. Radicais livres de oxigênio e exercício: mecanismos de formação e adaptação ao treinamento físico. Rev. Bras. Med. Esporte, v.10, p.308-318, 2004.

SIES, H.; STAHL, W. Vitamins E and C, $\alpha$ carotene, and other carotenoids as antioxidants. Am. J. Clin. Nutr., v.62, p.1315-21, 1995.

SILOTO, E.V.; ZEFERINO, C.P.; MOURA, A.S.A.M.T. et al. Temperatura e enriquecimento ambiental sobre o bem-estar de coelhos em crescimento. Cienc. Rural, v.39, p.528-533, 2009.

STOCKHAM, S. L.; SCOTT, M.A. Fundamentos de patologia clínica veterinária. Rio de Janeiro: Guanabara Koogan, 2011. 729p.

SZABO, C. Multiple pathways of peroxynitrite cytotoxicity. Toxicol. Lett, v.140-141, p.105-112, 2003.
VAN DE WEERD, H.A.; DOCKING, C.M.; DAY, J.E.L. et al. Effects of species-relevant environmental enrichment on the behaviour and productivity of finishing pigs. Appl. Anim. Behav. Sci., v.99, p.230-247, 2006.

VASCONCELOS, E.K.F.; BORGES, L.S.; SILVA, A.L. et al. Comportamento de suínos na fase de crescimento criados em ambiente enriquecido, J. Anim. Behav. Biometeorol., v.3, p.120-123, 2015.

VIEIRA, T.A.; CARVALHO, R.L.P.; FERREIRA, S.C. et al. The effects of environmental enrichment on biochemical and metric parameters of aged rats. Biosci. J., v.31, p.982-987, 2015.

ZWICKER, B.; GYGAX, L.; WECHSLER, B. et al. Short- and long-term effects of eight enrichment materials on the behaviour of finishing pigs fed ad libitum or restrictively. Appl. Anim. Behav. Sci., v.144, p.31-38, 2013. 